PROCEEDINGS OF THE

AMERICAN MATHEMATICAL SOCIETY

Volume 138, Number 6, June 2010, Pages 2079-2086

S 0002-9939(10)10265-2

Article electronically published on February 17, 2010

\title{
QUANTUM SPACES WITHOUT GROUP STRUCTURE
}

\author{
PIOTR M. SOŁTAN
}

(Communicated by Marius Junge)

\begin{abstract}
We prove that some well known compact quantum spaces such as quantum tori and some quantum two-spheres do not admit a compact quantum group structure. This is achieved by considering existence of traces, characters and nuclearity of the corresponding $\mathrm{C}^{*}$-algebras.
\end{abstract}

\section{INTRODUCTION}

Classical topology gives us many tools with which we can investigate whether a given topological space carries the structure of a topological group. In this paper we want to consider the same problem for non-commutative or quantum spaces. Not surprisingly some standard tricks from classical topology are not available in the non-commutative setting. As an example consider the standard exercise of showing that an interval with one or two end-points does not admit the structure of a topological group. The solution uses the fact that the neighborhoods of an end-point cannot be carried over homeomorphically onto neighborhoods of interior points of the interval whereas the hypothetical group law would provide such homeomorphisms. However, as of now, such arguments cannot be carried over to non-commutative topology.

Interestingly, however, many new tools which have no classical analog can be employed to study this problem for quantum spaces. Those used in this paper include studying existence of traces on the corresponding $\mathrm{C}^{*}$-algebras, the structure of the space of their characters and also properties such as nuclearity.

We will only consider compact quantum spaces, i.e. ones described by unital $\mathrm{C}^{*}$-algebras via the well established correspondence extending the Gelfand equivalence $(11,7)$. The main reasons for this are the very rich structure that compact quantum groups have and the ample literature on the subject (see e.g. [26, 1, 12]).

Before proceeding let us address a related problem. Recall that a compact quantum semigroup is a pair $\mathbb{S}=(A, \Delta)$ consisting of a unital $\mathrm{C}^{*}$-algebra $A$ and a comultiplication, i.e. a coassociative morphism $\Delta: A \rightarrow A \otimes A$ ([1, 20]). A compact quantum group is a compact quantum semigroup $\mathbb{G}=(A, \Delta)$ such that the sets

$$
\{\Delta(a)(\mathbb{1} \otimes b) \mid a, b \in A\} \quad \text { and } \quad\{(a \otimes \mathbb{1}) \Delta(b) \mid a, b \in A\}
$$

Received by the editors May 11, 2009, and, in revised form, September 21, 2009.

2000 Mathematics Subject Classification. Primary 46L89, 46L85, 17B37, 81R60, 20 G42.

Key words and phrases. Compact quantum group, quantum space, non-commutative topology.

This research was partially supported by a Polish government grant, no. N201 177033.

(C)2010 American Mathematical Society

Reverts to public domain 28 years from publication 
are linearly dense in $A \otimes A$ ([26, Definition 2.1]). In [26] an additional assumption of separability of $A$ was included, but thanks to the new proof of existence of Haar measure in 24] all results of 26] can be extended to the more general setting where $A$ is not assumed to be separable (see [12]).

The problem of whether a given quantum semigroup is in fact a compact quantum group can be quite difficult to approach directly. In 21] we showed that if $M$ is a finite dimensional $\mathrm{C}^{*}$-algebra, then the quantum semigroup $\mathbb{S}=(A, \Delta)$ of all self-maps of the finite quantum space underlying $M$ is not a compact quantum group unless $M=\mathbb{C}([21$, Proposition 2.1]). One must be careful here though. The simplest example, obtained by taking $M=\mathbb{C}^{2}$, leads to $A=\mathrm{C}^{*}\left(\mathbb{Z}_{2} * \mathbb{Z}_{2}\right)$, which, of course, does admit a compact quantum group structure but with a comultiplication different from $\Delta$.

A more explicit example was given in [21, Corollary 4.4], where we showed that the quantum commutant $(20,21])$ of a certain automorphism of $M_{2}$ is not a compact quantum group. In this example $A$ is the universal $\mathrm{C}^{*}$-algebra generated by three elements $\alpha, \beta$ and $\gamma$ such that $\beta=\beta^{*}, \gamma=\gamma^{*}$ and

$$
\begin{aligned}
\alpha^{*} \beta+\gamma \alpha^{*}+\alpha \gamma+\beta \alpha & =0, & \alpha^{2}+\beta \gamma=0, \\
\alpha \beta+\beta \alpha^{*} & =0, & \gamma \alpha+\alpha^{*} \gamma=0, \\
\alpha^{*} \alpha+\gamma^{2}+\alpha \alpha^{*}+\beta^{2} & =\mathbb{1}, &
\end{aligned}
$$

while the comultiplication $\Delta$ acts on generators in the following way:

$$
\begin{aligned}
& \Delta(\alpha)=\mathbb{1} \otimes \alpha+\left(\alpha^{*} \alpha+\gamma^{2}\right) \otimes\left(\alpha^{*}-\alpha\right)+\alpha \otimes \beta+\alpha^{*} \otimes \gamma, \\
& \Delta(\beta)=(\alpha \gamma+\beta \alpha) \otimes\left(\alpha-\alpha^{*}\right)+\beta \otimes \beta+\gamma \otimes \gamma, \\
& \Delta(\gamma)=(\beta \alpha+\alpha \gamma) \otimes\left(\alpha^{*}-\alpha\right)+\gamma \otimes \beta+\beta \otimes \gamma .
\end{aligned}
$$

The density conditions from the definition of a compact quantum group are difficult to check. However, using some advanced elements of the theory of compact quantum groups, we were able to prove that $\mathbb{S}=(A, \Delta)$ is not a compact quantum group.

In a similar context, in 22 we showed that the quantum semigroup of all maps of the quantum space underlying $M_{2}$ that preserve a certain degenerate state is not a compact quantum group. In this case $A$ is the universal $\mathrm{C}^{*}$-algebra generated by two elements $\beta$ and $\delta$ satisfying the relations

$$
\begin{aligned}
\beta \beta^{*} & =\mathbb{1}, \quad \delta^{2}=0, \\
\beta \delta & =0, \quad \beta \delta^{*}=0, \\
\beta^{*} \beta+\delta^{*} \delta+\delta \delta^{*} & =\mathbb{1},
\end{aligned}
$$

and the comultiplication $\Delta$ acts on generators in the following way:

$$
\begin{aligned}
& \Delta(\beta)=\beta \otimes \beta, \\
& \Delta(\delta)=\delta \otimes \beta+\beta^{*} \beta \otimes \delta+\delta^{*} \delta \otimes \delta .
\end{aligned}
$$

It is shown in [22, Proposition 5.5] that $\mathbb{S}=(A, \Delta)$ is not a quantum group.

In contrast to the cases dealt with above, in what follows we will not make any assumptions on the particular form of the comultiplication. We will show that the following compact quantum spaces do not admit any compact quantum group structure:

- all quantum tori,

- the standard Podleś sphere, 
- quantum two-spheres of Bratteli-Elliott-Evans-Kishimoto,

- Natsume-Olsen quantum spheres.

Quantum tori have been considered as non-commutative geometric objects in 6, 17. and numerous other papers. For surveys of and references on the remaining quantum spaces we recommend [8] as well as the original papers [15, 3, 4, 5, 13].

Let us now describe briefly the contents of the paper. In the next section we recall some basic elements and terminology of the theory of compact quantum groups. In Section 3 we formulate and prove the main results. This section is split into two subsections. The first deals with algebras with abundant traces while the other uses the fact that the $\mathrm{C}^{*}$-algebras under consideration have very few classical points.

\section{Preliminaries on COMPaCt Quantum groups}

Let $\mathbb{G}=(A, \Delta)$ be a compact quantum group. The $\mathrm{C}^{*}$-algebra $A$ is the algebra of continuous functions on $\mathbb{G}$. Theorem 2.2 of [26] tells us that there is a dense unital *subalgebra $\mathcal{A}$ in $A$ such that $\left.\Delta\right|_{\mathcal{A}}: \mathcal{A} \rightarrow \mathcal{A} \otimes_{\text {alg }} \mathcal{A}$ and that with this comultiplication $\mathcal{A}$ is a Hopf $*$-algebra. By [1, Theorem 5.1] this Hopf $*$-algebra is unique. The enveloping $\mathrm{C}^{*}$-algebra $A_{u}$ of $\mathcal{A}$ carries a comultiplication $\Delta_{u}: A_{u} \rightarrow A_{u} \otimes A_{u}$ such that $\mathbb{G}_{u}=\left(A_{u}, \Delta_{u}\right)$ is a compact quantum group. Note that $\mathcal{A}$ is then naturally a dense subalgebra of $A_{u}$.

There is a unique epimorphism $\rho: A_{u} \rightarrow A$ extending the identity map on $\mathcal{A}$ and it satisfies $(\rho \otimes \rho) \circ \Delta_{u}=\Delta \circ \rho$. The compact quantum group $\mathbb{G}_{u}$ is called the universal version of $\mathbb{G}$. We will often use the fact that any $*$-character of $\mathcal{A}$ extends continuously to $A_{u}$ ([1, Theorem 3.6]).

The famous theorem of Woronowicz ([25, Theorem 4.2], [26, Theorem 2.3]) says that if $\mathbb{G}=(A, \Delta)$ is a compact quantum group, then there exists a unique state $h$ on $A$ such that

$$
(h \otimes \mathrm{id}) \Delta(a)=(\mathrm{id} \otimes h) \Delta(a)=h(a) \mathbb{1}
$$

for any $a \in A$. This state is called the Haar measure of $\mathbb{G}$. The state $h$ may not be faithful, but it is known ([25, page 656]; see also 11, Theorem 2.1]) that the ideal $\mathcal{J}=\left\{a \in A \mid h\left(a^{*} a\right)=0\right\}$ is two-sided. Let $A_{r}=A / \mathcal{J}$ and denote by $\lambda$ the quotient map $A \rightarrow A_{r}$. The map $\lambda$ is injective on the dense subalgebra $\mathcal{A}$, so $A_{r}$ can be viewed as a different completion of $\mathcal{A}$. It can be shown that there is a comultiplication $\Delta_{r}: A_{r} \rightarrow A_{r} \otimes A_{r}$ extending that of $\mathcal{A}$ such that $\mathbb{G}_{r}=\left(A_{r}, \Delta_{r}\right)$ is a compact quantum group called the reduced version of $\mathbb{G}$. We have $(\lambda \otimes \lambda) \circ \Delta=\Delta_{r} \circ \lambda$. Moreover the Haar measure $h_{r}$ of $\mathbb{G}_{r}$ is faithful and $h=h_{r} \circ \lambda$ (similarly the Haar measure $h_{u}$ of $\mathbb{G}_{u}$ satisfies $h_{u}=h \circ \rho$ ).

To illustrate the concepts dealt with above, take $A$ to be a $\mathrm{C}^{*}$-completion of the group algebra $\mathbb{C}[\Gamma]$ of a discrete group $\Gamma$ such that the comultiplication $\gamma \mapsto \gamma \otimes \gamma$ (for $\gamma \in \Gamma$ ) extends to a $*$-homomorphism $\Delta: A \rightarrow A \otimes A$. Then $\mathbb{G}=(A, \Delta)$ is a compact quantum group. The $\mathrm{C}^{*}$-algebras $A_{u}$ and $A_{r}$ are $\mathrm{C}^{*}(\Gamma)$ and $\mathrm{C}_{r}^{*}(\Gamma)$. The Haar measure on $\mathbb{G}_{r}$ is the von Neumann trace on $\mathrm{C}_{r}^{*}(\Gamma)$. Clearly if $\Gamma$ is amenable, then $A, A_{u}$ and $A_{r}$ are canonically isomorphic. Note that the example $\mathbb{G}=(A, \Delta)$ with $A=\mathrm{C}_{r}^{*}\left(\mathbb{F}_{2}\right)$ shows that the algebra of continuous functions on a compact quantum group may be simple.

Now let $\mathbb{G}=(A, \Delta)$ be a compact quantum group. The special situation where $\rho$ and $\lambda$ are isomorphisms is called co-amenability of $\mathbb{G}$. There are various equivalent definitions of this concept and we will use some of them. For details we refer to 11. In particular if $\mathbb{G}$ is co-amenable, then the co-unit $e$ of $\mathcal{A}$ extends to a 
character of $A$ (because $A=A_{u}$ ) and this extension (also denoted by $e$ ) still satisfies $(e \otimes \mathrm{id}) \circ \Delta=(\mathrm{id} \otimes e) \circ \Delta=\mathrm{id}$. One can easily see that if any of the $\mathrm{C}^{*}$-algebras $A, A_{u}$ or $A_{r}$ is commutative, then they are all the same and $\mathbb{G}$ is co-amenable.

The Haar measure $h$ of $\mathbb{G}$ need not be a trace. Whenever $h$ is a trace we say that $\mathbb{G}$ is of Kac type. Groups of Kac type can be characterized in many different ways (see e.g. [26, Theorem 2.5]). The study of compact quantum groups which are not of Kac type led to the discovery that there always exists a certain family $\left(f_{z}\right)_{z} \in \mathbb{C}$ of multiplicative functionals on $\mathcal{A}$ which encodes modular properties of $h$. We always have $f_{0}=e$ (the co-unit), and the family is trivial (i.e. $f_{z}=f_{0}$ for all $z$ ) if and only if $\mathbb{G}$ is of Kac type ([26, Theorem 2.5(2)]). Moreover this family is holomorphic in the sense that for any $a \in \mathcal{A}$ the function $z \mapsto f_{z}(a)$ is entire. For $z$ on the imaginary axis, the $f_{z}$ are $*$-characters and consequently extend to characters of $A_{u}$. We call these functionals the Woronowicz characters of $\mathbb{G}$.

\section{NON-EXISTENCE OF COMPACT QUANTUM GROUP STRUCTURE}

3.1. Algebras with abundant traces. We will first recall a result formulated in [19, Remark A.2].

Theorem 3.1. Let $\mathbb{G}=(A, \Delta)$ be a compact quantum group. Assume that for any $a \in A$ there exists a tracial state $\tau$ on $A$ such that $\tau\left(a^{*} a\right) \neq 0$. Then $\mathbb{G}$ is of Kac type.

In the proof of the next corollary we will use known facts about rotation algebras $A_{\theta}([16,17])$.

Corollary 3.2. For any $\theta \in] 0,1\left[\right.$ the rotation algebra $A_{\theta}$ does not admit a compact quantum group structure.

Proof. The $\mathrm{C}^{*}$-algebra $A_{\theta}$ admits a faithful trace (unique if $\theta$ is irrational). Therefore if there were to exist a comultiplication $\Delta: A_{\theta} \rightarrow A_{\theta} \otimes A_{\theta}$ such that $\mathbb{G}=$ $\left(A_{\theta}, \Delta\right)$ is a compact quantum group, the Haar measure on $\mathbb{G}$ would be a trace. It is well known that $A_{\theta}$ is a nuclear $\mathrm{C}^{*}$-algebra (e.g. because it is a crossed product of a commutative algebra by an action of an amenable - in fact commutative group). By [2, Theorem 1.1] (see also [18, 23]) the compact quantum group $\mathbb{G}$ must be co-amenable. In particular the $\mathrm{C}^{*}$-algebra $A_{\theta}$ would have to admit a continuous co-unit. Clearly this is not the case, since for $\theta$ irrational $A_{\theta}$ is simple and for rational $\theta$ we have $A_{\theta} \cong M_{N} \otimes \mathrm{C}(\mathbb{T})$, which also does not admit a character.

It is interesting to note that if $\theta$ is irrational, the von Neumann algebra we obtain from $A_{\theta}$ via the GNS construction is the hyperfinite factor of type $\mathrm{II}_{1}$. This von Neumann algebra is a von Neumann algebra of measurable functions on a compact quantum group. For example it is the group von Neumann algebra of any discrete amenable i.c.c. group. Moreover, as shown in [10] the quantum two-torus can be a "part" of a compact quantum group (namely the quantum double torus).

Let us summarize the argument used to prove that quantum 2-tori are not quantum groups in the following statement:

Theorem 3.3. Let $A$ be a nuclear unital $\mathrm{C}^{*}$-algebra having a faithful family of tracial states and not admitting a continuous character. Then there does not exist a comultiplication $\Delta: A \rightarrow A \otimes A$ such that $(A, \Delta)$ is a compact quantum group. 
It follows that the Bratteli-Elliott-Evans-Kishimoto quantum spheres ( 3 , 4, 5, 8, $)$ do not admit a quantum group structure. The algebra $C_{\theta}$ of continuous functions on the BEEK two-sphere for the deformation parameter $\theta$ is the fixed point subalgebra of $A_{\theta}$ under the action of $\mathbb{Z}_{2}$ mapping $u$ and $v$ to their adjoints. This $\mathrm{C}^{*}$-algebra is nuclear and possesses a faithful trace. Also $C_{\theta}$ does not admit a character.

The same reasoning now shows that higher dimensional quantum tori ([17]) do not admit a compact quantum group structure. They do not have characters, have a faithful trace (often unique) and are nuclear. Note also that Theorem 3.3 rules out many AF-algebras because they are nuclear, admit traces and often do not admit characters (like e.g. all UHF-algebras).

3.2. Algebras admitting few characters. In Section 3.1 we showed that some $\mathrm{C}^{*}$-algebras do not admit a compact quantum group structure by using the fact that they do not possess a character. However, in some situations, we can use the existence of a character on a $\mathrm{C}^{*}$-algebra to prove that it cannot be endowed with a comultiplication making it a compact quantum group. In the cases we consider it is possible to prove that should the quantum space under consideration be a compact quantum group, then the Haar measure cannot be a trace. This, together with co-amenability, guarantees the existence of many characters, which we know is not the case.

In the case of the quantum two-spheres of Podleś (for the standard quantum sphere, see [15, 8) and Natsume-Olsen $(13,8)$ ) our reasoning will be based on the following theorem of Bedos, Murphy and Tuset:

Theorem 3.4 ([1, Theorem 2.8]). Let $\mathbb{G}=(A, \Delta)$ be a compact quantum group such that its Haar measure is faithful. Then $\mathbb{G}$ is co-amenable if and only if $A$ admits a character.

Using this we have:

Theorem 3.5. The standard Podleś quantum sphere $S_{q, 0}^{2}$ is not a compact quantum group.

Proof. The $\mathrm{C}^{*}$-algebra of continuous functions on the standard Podleś quantum sphere is isomorphic to $\mathcal{K}^{+}$, i.e. the minimal unitization of the algebra $\mathcal{K}$ of compact operators on a separable Hilbert space. Assume that $\Delta: \mathcal{K}^{+} \rightarrow \mathcal{K}^{+} \otimes \mathcal{K}^{+}$is a comultiplication making $\mathbb{G}=\left(\mathcal{K}^{+}, \Delta\right)$ a compact quantum group. Then, first of all, the Haar measure of $\mathbb{G}$ must be faithful. This is because if it were not faithful, then the $\mathrm{C}^{*}$-algebra of continuous functions on the reduced compact quantum group $\mathbb{G}_{r}=\left(A_{r}, \Delta_{r}\right)$ would be one dimensional and in particular commutative. As we pointed out in Section 2, commutative $A_{r}$ means that $\mathbb{G}$ is co-amenable and that $\mathbb{G}_{r}=\mathbb{G}$.

Therefore the Haar measure on $\mathbb{G}$ is faithful. However, $\mathcal{K}^{+}$clearly admits a character, so $\mathbb{G}$ must be co-amenable by Theorem 3.4. This means that $\mathbb{G}$ is at the same time universal, and so $\mathcal{K}^{+}$is the enveloping $\mathrm{C}^{*}$-algebra of the Hopf *algebra $\mathcal{A}$ canonically associated to $\mathbb{G}$. Now let $\left(f_{z}\right)_{z \in \mathbb{C}}$ be the family of modular functionals on $\mathcal{A}$ ([26, Theorem 2.4]). Then $\left(f_{i t}\right)_{t \in \mathbb{R}}$ is a family of $*$-characters of $\mathcal{A}$, so all these elements must be continuous on the universal completion $\mathcal{K}^{+}$of $\mathcal{A}$. However there is only one character on $\mathcal{K}^{+}$, so $f_{i t}=f_{0}$ for all $t \in \mathbb{R}$. By the fact that $\left(f_{z}\right)_{z \in \mathbb{C}}$ is a holomorphic family ([26, Theorem 2.4(2)]) we see that the whole family is trivial, namely $f_{z}=f_{0}$ for all $z \in \mathbb{C}$. By [26, Theorem 2.5] the Haar measure of $\mathbb{G}$ is a trace, but there is no faithful trace on $\mathcal{K}^{+}$. 
We will now show that the Natsume-Olsen quantum spheres cannot be endowed with a compact quantum group structure.

Theorem 3.6. The Natsume-Olsen quantum sphere $S_{t}^{2}$ is not a compact quantum group for any $t \in\left[0, \frac{1}{2}[\right.$.

Proof. The family of Natsume quantum spheres consists really of only two elements. For the deformation parameter $t=0$ we obtain the classical two-sphere, which is not a compact group, while for $t \in] 0, \frac{1}{2}$ [ the $\mathrm{C}^{*}$-algebras describing the Natsume quantum sphere are all isomorphic to a fixed $\mathrm{C}^{*}$-algebra $B$. What we will need now is that $B$ fits into the exact sequence

$$
0 \longrightarrow \mathcal{K} \otimes \mathrm{C}(\mathbb{T}) \longrightarrow B \stackrel{\pi}{\longrightarrow} \mathbb{C}^{2} \longrightarrow 0
$$

([13, Proposition $3.1 \&$ Corollary 3.2]). If we assume that there exists $\Delta: B \rightarrow$ $B \otimes B$ such that $\mathbb{G}=(B, \Delta)$ is a compact quantum group, then the Haar measure $h$ of $\mathbb{G}$ cannot be a trace. Indeed, note that for any $x$ in the ideal $\mathcal{I}=\mathcal{K} \otimes \mathrm{C}(\mathbb{T})=$ ker $\pi \subset B$ and any trace $\tau$ on $B$ we have $\tau(x)=01$ In particular if $h$ were a trace, then $h\left(x^{*} x\right)=0$ for all $x \in \mathcal{I}$. Therefore if $\mathbb{G}_{r}=\left(B_{r}, \Delta_{r}\right)$ is the reduced quantum group, the ideal $\mathcal{J}$ such that $B_{r}=B / \mathcal{J}$ (i.e. the left kernel of $h$ ) contains $\mathcal{I}$. But this means that $B_{r}$ is a quotient of $B / \mathcal{I}=\mathbb{C}^{2}$ which is commutative. As we argued in the proof of Theorem 3.5 this is not possible since compact quantum groups with commutative $\mathrm{C}^{*}$-algebras are universal.

We will now show that $B_{r}$ possesses a character. Recall ([13, Definition 1.7 $\&$ Proposition 3.5]) that $B$ is generated by two elements $z$ and $\zeta$ satisfying the following relations:

$$
\begin{gathered}
\zeta^{*} \zeta+z^{2}=\mathbb{1}=\zeta \zeta^{*}+\left(t \zeta \zeta^{*}+z\right)^{2}, \\
\zeta z-z \zeta=t \zeta\left(\mathbb{1}-z^{2}\right),
\end{gathered}
$$

where $t$ is a fixed number from $] 0, \frac{1}{2}[$. Note that $z$ cannot belong to any proper ideal of $B$. Indeed, it follows from (3.1) that

$$
\zeta=\frac{1}{t}\left(\zeta z-z \zeta+t z^{2}\right)
$$

belongs to any ideal to which $z$ belongs and thus any such ideal must be $B$. Therefore $z$ does not belong either to $\mathcal{I}$ or to $\mathcal{J}$.

The possibility that $B=\mathcal{I}+\mathcal{J}$ must be ruled out because we would then have by [9, Corollary 1.8.4] that

$$
B_{r}=B / \mathcal{J}=(\mathcal{I}+\mathcal{J}) / \mathcal{J}=\mathcal{I} /(\mathcal{I} \cap \mathcal{J}) \cong \mathcal{K} \otimes \mathrm{C}(X)
$$

where $X$ is a compact space (it is a closed subset of $\mathbb{T}$ because $\mathcal{I} \cap \mathcal{J}$ is an ideal in $\mathcal{I}=\mathcal{K} \otimes \mathrm{C}(\mathbb{T})$, so it must be of the form $\mathcal{K} \otimes \mathcal{R}$ where $\mathcal{R}$ is an ideal of $\mathrm{C}(\mathbb{T})$ ). This, however, cannot happen because the last algebra on the right hand side of (3.2) does not have a unit.

\footnotetext{
${ }^{1}$ For any simple tensors $k_{1} \otimes f_{1}, k_{2} \otimes f_{2} \in \mathcal{K} \otimes \mathrm{C}(\mathbb{T})$ we have $\tau\left(k_{1} k_{2} \otimes f_{1} f_{2}\right)=\tau\left(k_{2} k_{1} \otimes f_{2} f_{1}\right)=$ $\tau\left(k_{2} k_{1} \otimes f_{1} f_{2}\right)$. Therefore $\tau(k \otimes f)=0$ for any $f \in \mathrm{C}(\mathbb{T})$ and any $k \in \mathcal{K}$ which is a finite sum of commutators. From [14, Theorem 1] we know that these are all compact operators, so $\tau=0$ on $\mathcal{K} \otimes \mathrm{C}(\mathbb{T})$. Equivalently we can use the simple fact already used in [13] that $\mathcal{K} \otimes \mathrm{C}(\mathbb{T})$ is the crossed product $\mathrm{C}_{0}(\mathbb{R}) \rtimes_{\alpha} \mathbb{Z}$, where $\alpha$ is translation by one. Clearly there is no invariant probability measure on $\mathbb{R}$ for this action.
} 
Therefore $z \notin(\mathcal{I}+\mathcal{J})$, and so, denoting by $\lambda$ the quotient map $B \rightarrow B_{r}=B / \mathcal{J}$, we have that $\lambda(z) \notin \lambda(\mathcal{I})$. In particular $\lambda(\mathcal{I})$ is a proper ideal of $B_{r}$. The (nonzero) quotient

$$
B_{r} / \lambda(\mathcal{I})
$$

is then isomorphic to $B /(\mathcal{I}+\mathcal{J})$, which in turn is isomorphic to a quotient of $B / \mathcal{I}=\mathbb{C}^{2}$. This algebra clearly has at least one character.

Since $B_{r}$ has a character, we have by Theorem 3.4 that $\mathbb{G}_{r}$ is co-amenable and consequently that $\lambda$ is an isomorphism and $\mathbb{G}=\mathbb{G}_{r}=\mathbb{G}_{u}$. In particular the Woronowicz characters $\left(f_{i t}\right)_{t \in \mathbb{R}}$ of $\mathbb{G}$ must be continuous on $B$. However, since $h$ is not a trace, the family $\left(f_{i t}\right)_{t \in \mathbb{R}}$ must be a nontrivial continuous family of characters. This stands in contradiction with the fact that the space of characters of $B$ is a discrete two-point space.

\section{REFERENCES}

1. E. Bedos, G. Murphy and L. Tuset, Co-amenability of compact quantum groups. J. Geom. Phys. 40 (2001), 129-153. MR 1862084 (2002m:46100)

2. E. Bedos, G. Murphy and L. Tuset, Amenability and co-amenability of algebraic quantum groups. II. J. Funct. Anal. 201 (2003), 303-340. MR.1986692 (2004e:46085)

3. O. Bratteli, G.A. Elliott, D.E. Evans and A. Kishimoto, Non-commutative spheres. I. Int. J. Math. 3 (1991), 139-166. MR1094701(92d:58008)

4. O. Bratteli, G.A. Elliott, D.E. Evans and A. Kishimoto, Non-commutative spheres. II. Rational rotations. J. Op. Th. 27 (1992), 53-85. MR.1241115 (94j:46068)

5. O. Bratteli and A. Kishimoto, Non-commutative spheres. III. Irrational rotations. Comm. Math. Phys. 147 (1992), 605-624. MR1175495 (93g:58008)

6. A. Connes, $C^{*}$-algebrès et géométrie différentielle. C. R. Acad. Sci. Paris Sér. A-B, 290 (1980), 599-604. MR572645 (81c:46053)

7. A. Connes, Noncommutative Geometry. Academic Press, 1994. MR.1303779 (95j:46063)

8. L. Dąbrowski, The garden of quantum spheres. In Noncommutative Geometry and Quantum Groups, Banach Center Publications, 61, Polish Academy of Sciences, 2003, 37-48. MR.2024420 (2004k:58009)

9. J. Dixmier, $C^{*}$-algebras. North-Holland, 1977. MR0458185 (56:16388)

10. P.M. Hajac and T. Masuda, Quantum double torus. C. R. Acad. Sci. Paris Ser. I Math. 327, no. 6 (1998), 553-558. MR1650603 (99h:16060)

11. P. Kruszyśki and S.L. Woronowicz, A non-commutative Gelfand-Naimark theorem. J. Op. Th. 8 (1982), 361-389. MR677419 (84b:46068)

12. A. Maes and A. Van Daele, Notes on compact quantum groups. Nieuw Arch. Wiskd. IV 16, no. 1-2 (1998), 73-112. MR.1645264 (99g:46105)

13. T. Natsume and C.L. Olsen, A new family of noncommutative 2-spheres. J. Func. Anal. 202 (2003), 363-391. MR1990530 (2005f:46126)

14. C. Pearcy and D. Topping, On commutators in ideals of compact operators. Michigan Math. J. 18, no. 3 (1971), 247-252. MR0284853 (44:2077)

15. P. Podleś, Quantum spheres. Lett. Math. Phys. 14 (1987), 193-202. MR919322 (89b:46081)

16. M.A. Rieffel, Non-commutative tori - a case study of non-commutative differentiable manifolds. Contemporary Mathematics, 105, Amer. Math. Soc., Providence, RI, 1990, 191-211. MR.1047281 (91d:58012)

17. M.A. Rieffel, C*-algebras associated to irrational rotations. Pac. J. Math. 93 (1981), 415-429. MR623572(83b:46087)

18. Z.-J. Ruan, Amenability of Hopf von Neumann algebras and Kac algebras. J. Funct. Anal. 139 (1996), 466-499. MR.1402773 (98e:46077)

19. P.M. Soltan, Quantum Bohr compactification. Ill. J. Math. 49, no. 4 (2005), 1245-1270. MR2210362 (2007a:46079)

20. P.M. Soltan, Quantum families of maps and quantum semigroups on finite quantum spaces. J. Geom. Phys. 59 (2009), 354-368. MR 2501746

21. P.M. Soltan, Examples of quantum commutants. Arab. J. Sci. Eng. 33, no. 2C (2008), 447457. MR2500052 
22. P.M. Soltan, Quantum $\mathrm{SO}(3)$ groups and quantum group actions on $M_{2}$. J. Noncommut. Geom. 4 (2010), 2-28.

23. R. Tomatsu, Amenable discrete quantum groups. J. Math. Soc. Japan 58 (2006), 949-964. MR2276175 (2008g:46122)

24. A. Van Daele, The Haar measure on a compact quantum group. Proc. Amer. Math. Soc. 123 (1995), 3125-3128. MR1277138 (95m:46097)

25. S.L. Woronowicz, Compact matrix pseudogroups. Comm. Math. Phys. 111 (1987), 613-665. MR $901157(88 \mathrm{~m}: 46079)$

26. S.L. Woronowicz, Compact quantum groups. In Symétries Quantiques, les Houches, Session LXIV 1995, Elsevier, 1998, 845-884. MR.1616348 (99m:46164)

Institute of Mathematics, Polish Academy of Sciences - And - Department of Mathematical Methods in Physics, Faculty of Physics, University of Warsaw, Warsaw, Poland E-mail address: piotr.soltan@fuw.edu.pl 